Scientific Journal of Hamadan Nursing \& Midwifery Faculty - ISSN 2008-2819

\title{
The Study of the Impact of Model Based Education on Midlife Women's Physical Activity in Hamadan
}

\section{Shohreh Emdadi', Seyed Mohammad Mehdi Hazavehei ${ }^{*}$, Alireza Soltanian ${ }^{3}$, Saeed Bashirian $^{4}$, Rashid Heidari Moghadam ${ }^{5}$, Maryam Farhadian', Majid Barati ${ }^{4}$}

1. PhD Student of Health Education \& Promotion, Student Research Committee, Hamadan University of Medical Sciences, Hamadan, Iran

2. Health Sciences Research Center, Department of Public Health, School of Public Health, Hamadan University of Medical Sciences, Hamadan, Iran

3. Modeling of Non-Communicable Disease Research Center, Hamadan University of Medical Sciences, Hamadan, Iran

4. Social Determinants of Health Research Center, Hamadan University of Medical Sciences, Hamadan, Iran

5. Department of Ergonomics, School of Public Health, Research Center for Health Sciences, Hamadan University of Medical Sciences, Hamadan, Iran

6. Modeling of Non-Communicable Disease Research Center, Hamadan University of Medical Sciences, Hamadan, Iran

\begin{tabular}{lr}
\hline \multicolumn{2}{c}{ Article Info } \\
\hline & \\
Received: & $2017 / 01 / 21$ \\
Accepted: & $2017 / 02 / 19$ \\
Published Online & $2017 / 07 / 17$
\end{tabular}

DOI: $10.30699 /$ sjhnmf.26.5.1

Original Article

Use your device to scan and read the article online

\section{Abstract}

Introduction: Regular and daily physical activity delays the midlife and menopausal consequences and promotes the women's quality of life. Physical activity increases management of diabetes, cardiovascular disease, osteoporosis and high blood pressure. Purpose of this study is to determine the impact of model based education on midlife women's physical activity in Hamadan.

Methods: 122 midlife women 50 to 64 years old chosen by random sampling participated in this experimental study. The questionnaire was designed by researcher and measured the model structures. Pedometer was utilized to measure walking and BMI was measured as physical activity consequences.

Results: After three months, evaluation showed significant changes in PRECEDE model structures $(P<0 / 001)$. Walking increased $(P<0 / 001)$. BMI decreased significantly $(P<0 / 001)$.

Conclusion: Changes were observed in structures based on PRECEDE model. Walking showed successfulness for midlife women. Reducing BMI was observed.

Keywords: Educational Program, Physical Activity, PRECEDE Model, Midlife Women, Pedometer.

How to Cite This Article:

Emdadi S, Hazavehei S M M, Soltanian A, Bashirian S, Heidari Moghadam R, Farhadian M et al . The Study of the Impact of Model Based Education on Midlife Women's Physical Activity in Hamadan. Sci J Hamadan Nurs Midwifery Fac. 2018; 25 (5): 154 - 162 


\section{بررسى تأثير آموزش مبتنى بر مدل بر فعاليت جسمانى زنان ميانسال شهر همدان}

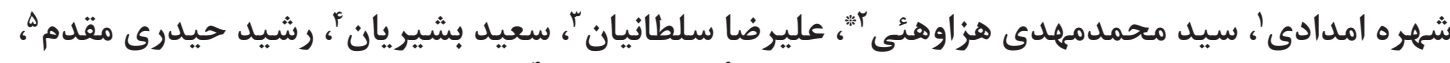

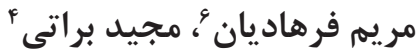

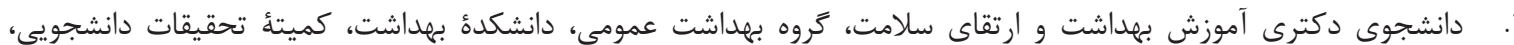

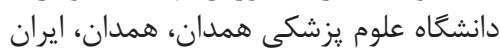

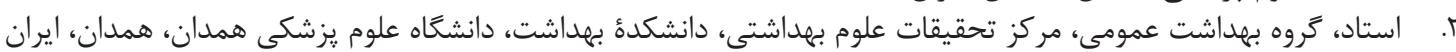

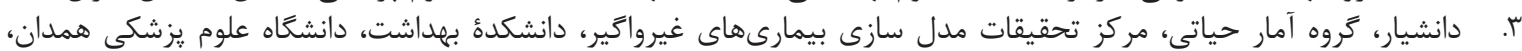

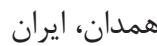
F. استاديار، مركز تحقيقات عوامل اجتماعى مؤثر بر سلامت، كروه بهداشت عمومى، دانشكدة بهداشت، دانشكاه علوم يزشكى همدان،

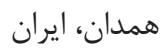

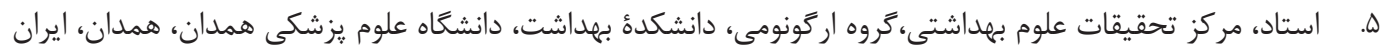

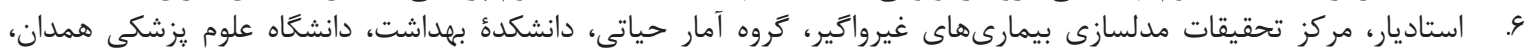
همدان، ايران

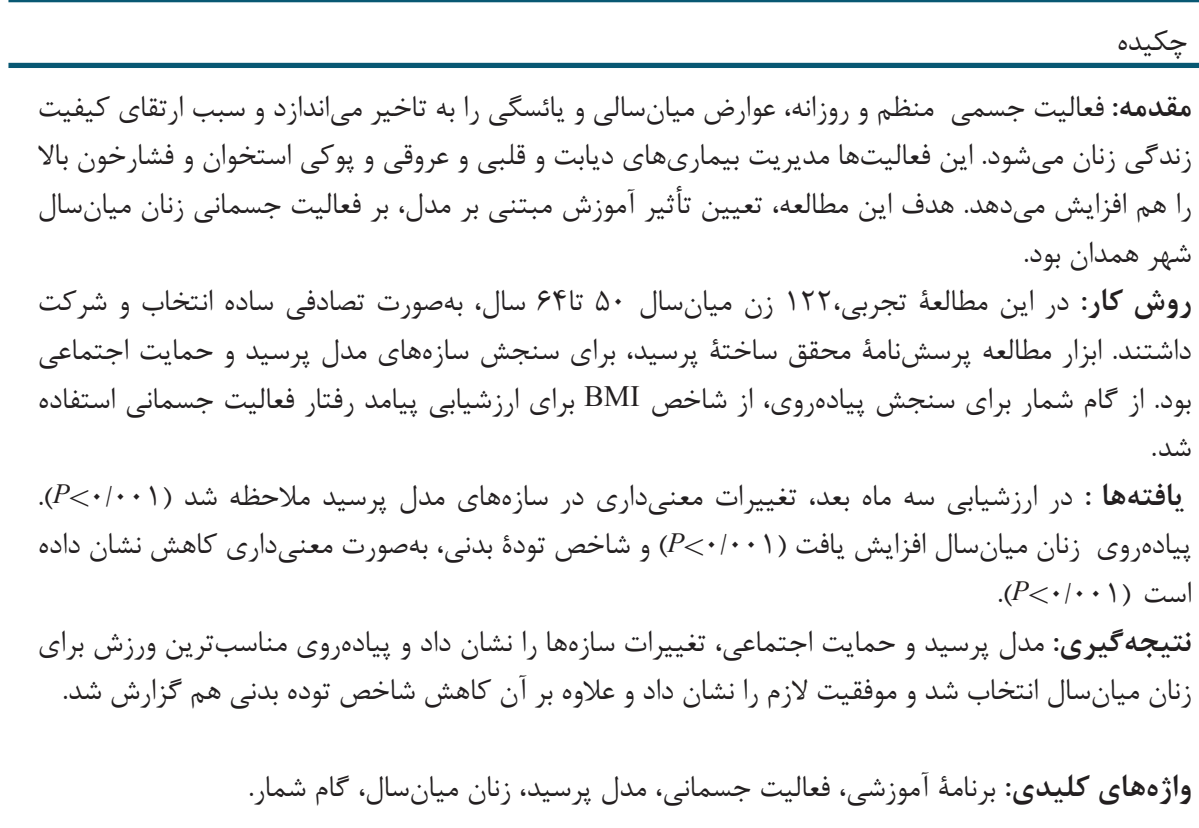

\section{جكيده}

政

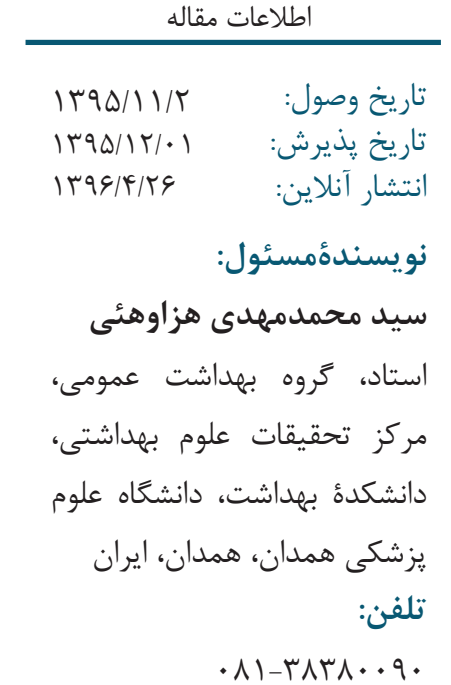

$\cdot \wedge 1-r \wedge r \Lambda \cdot .9$.
مى گذارد بلكه منجر به مرى ايشان مىشود. افزايش وزن،

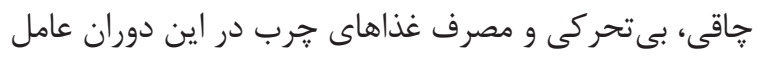

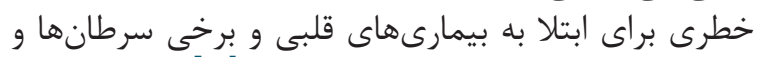

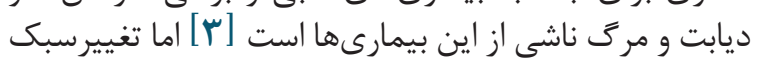

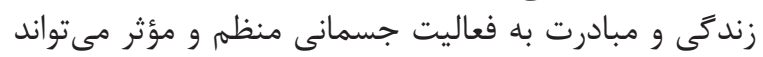

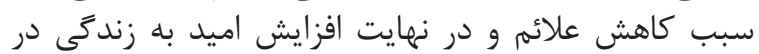

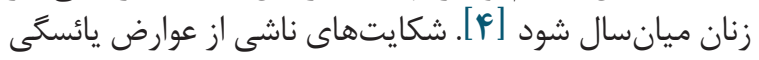

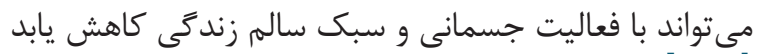

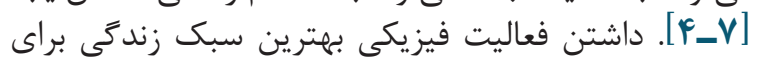

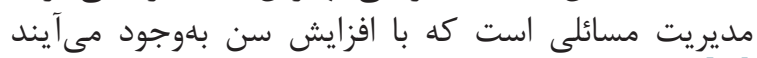

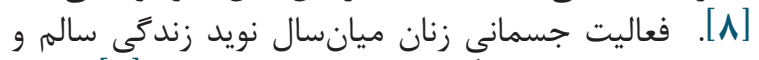

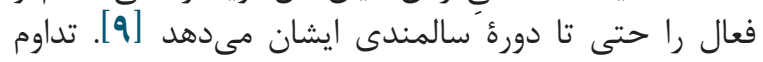

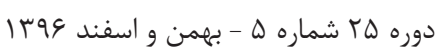

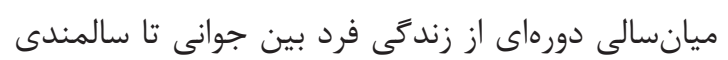

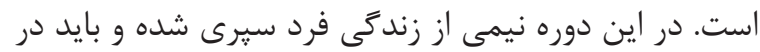

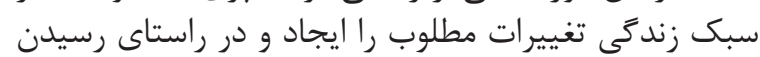

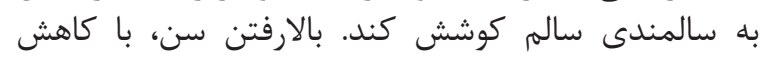

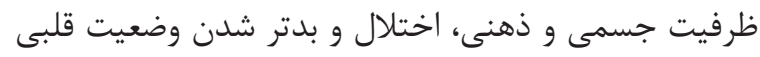

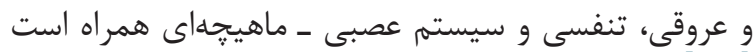

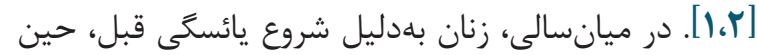

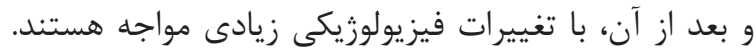

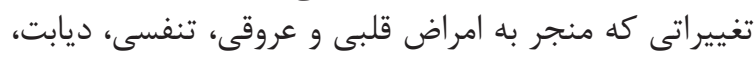

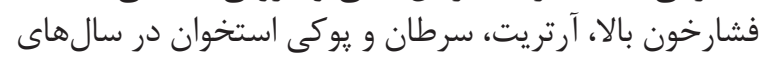

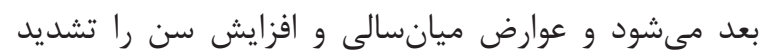

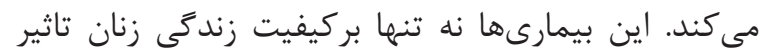

$$
\text { مجله علمى دانشكده يرستارى و مامايى همدان }
$$


برهانى ديزجى و همكاران سال سوجاتادر مينو دشت،

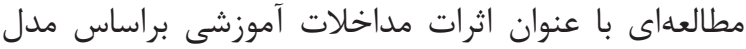

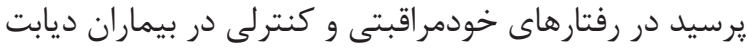

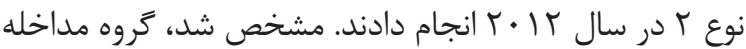

توانسته رفتارهاى خود مراقبتى را افزايش دهان مهد [19]. اين مطالعه با هدف، تعيين تاثير آموزش براساس مدل

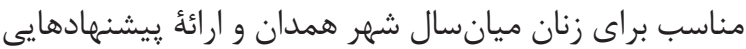

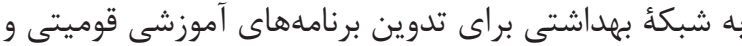

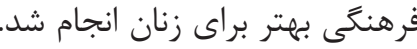

\section{روش كار}

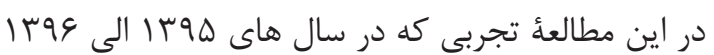

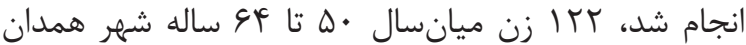

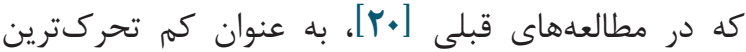

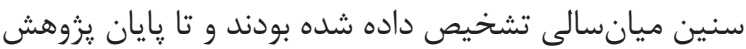

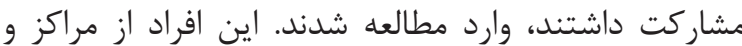

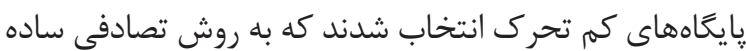

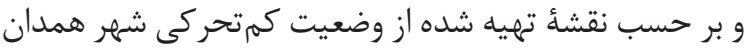
در مطالعههاى قبلى مشخص شد شد [ب.

معيارهاى ورود به مطالعه از اين قرار بود: زن بودن،

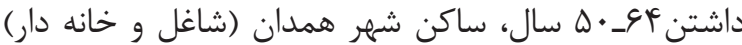

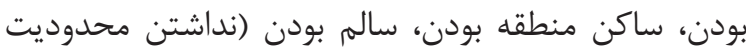

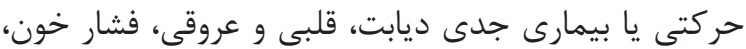

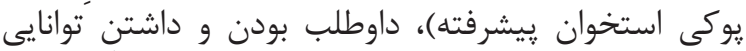

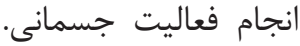

حجم نمونه

$$
n=\frac{2\left(z_{1-\frac{\alpha}{2}}+z_{1-\beta}\right) \delta^{2}}{d^{2}}+(م \text { ) }
$$

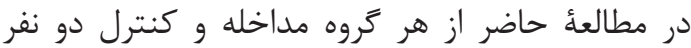

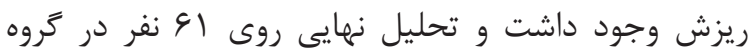

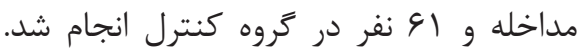

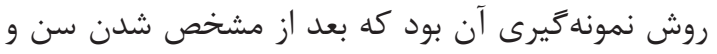

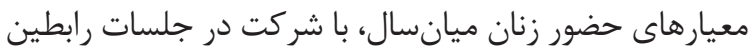

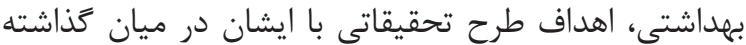

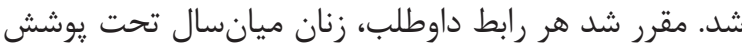

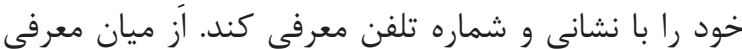

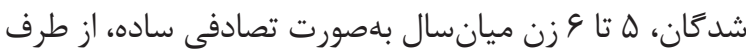

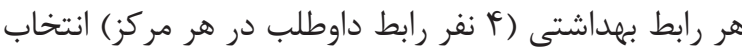

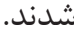

براى اندازهيرى مشخصات دموكرافيك و اندازهزيرى

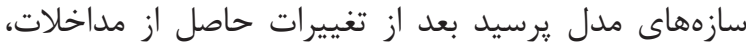

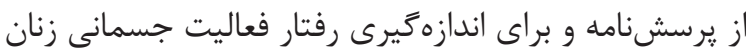
ميانسال شهر همدان از كام شمار استفاده شدئ

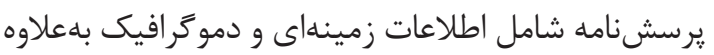

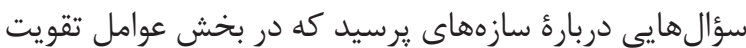

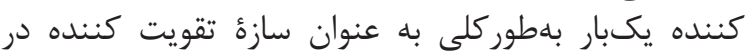

فعاليت جسمانى منظم روزانه از توسعه و افزايش ناتوانىهاى

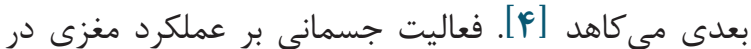

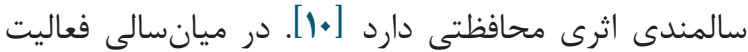

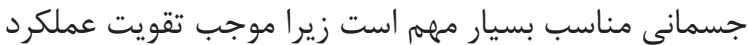

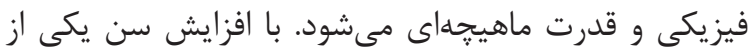

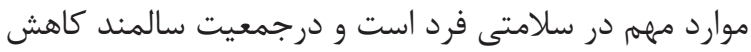

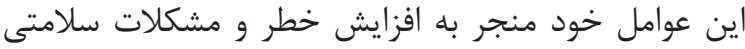

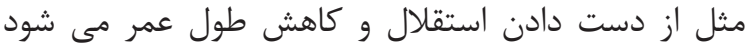

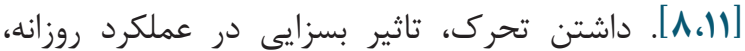

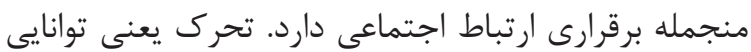

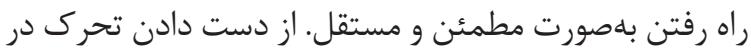

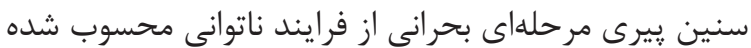

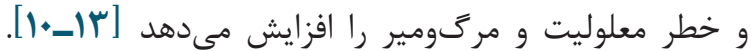

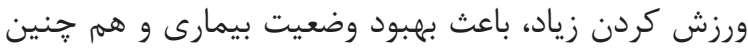
موجب بيشخيرى از آسيب عوامل مضر بر سلامتى مى شئود. بر طبق كزارشهاى سازمانهاى جهانى بهداشت كمتر

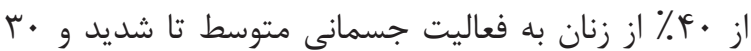

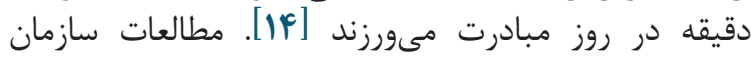

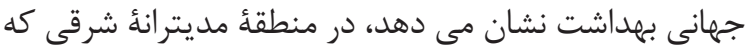

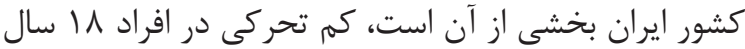

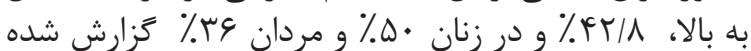

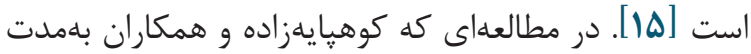

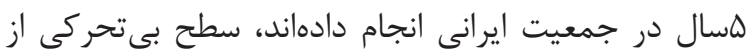

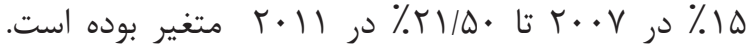
بلهويزه زنان كم تحركتر در شناخته

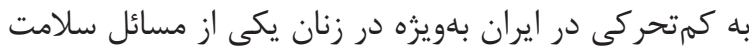

عمومى ذكر شده در است [19].

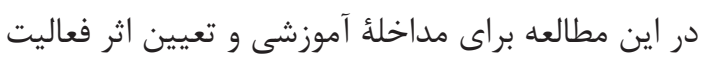

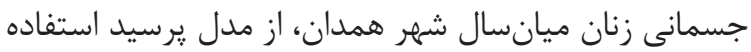

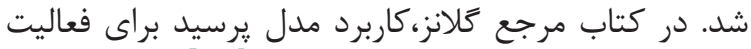

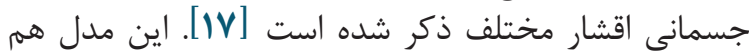

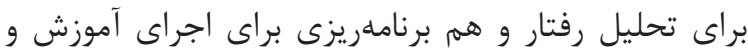

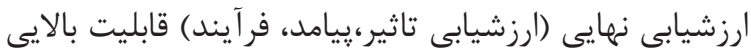

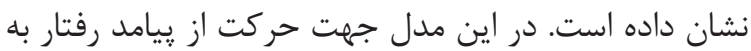

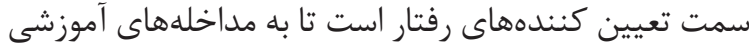

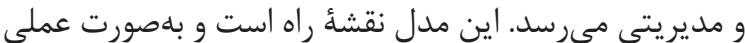

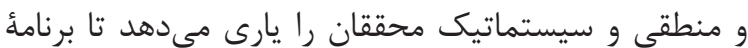

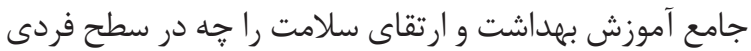

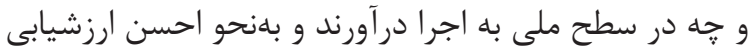

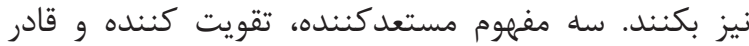
كننده از مفاهيم عمده در يرسيد هستند [IV]

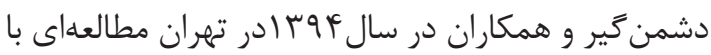

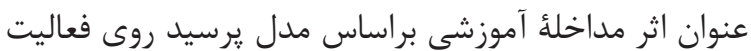

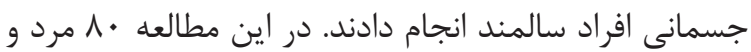

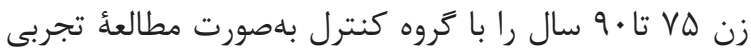

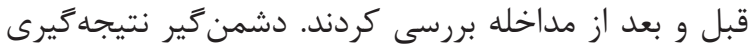

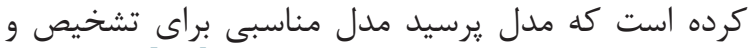
اجراى برنامههاى آموزشى سالمندان است [11] 
همسائُ يكديگر بودند و در بيشتر مراسم اجتماعى به يارى ون و

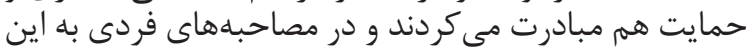

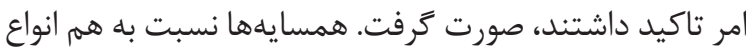

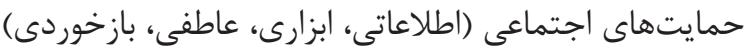

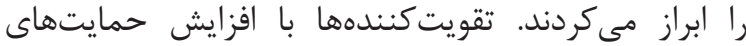

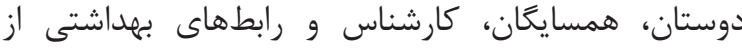
خانواده به سمت فراهم كنند

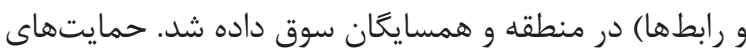

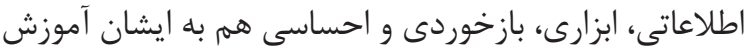
داده شد.

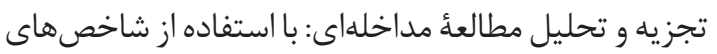
مركزى و يراكند

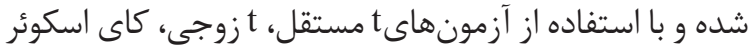

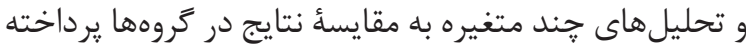

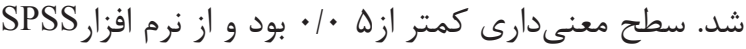

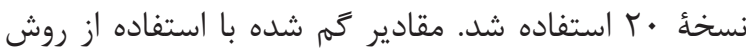
برot-deck تحليل انجام شد.

\section{كافتهها}

يافتهها نشان داد، ميانگين سنى گروه مداخله

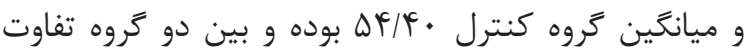

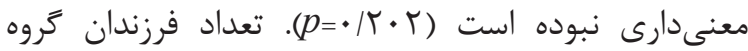

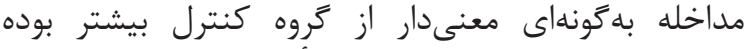

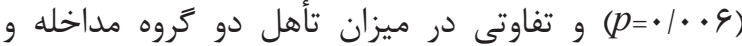

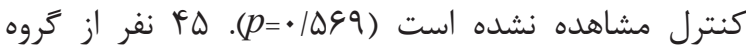

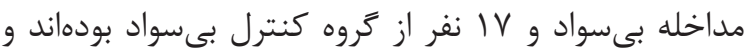

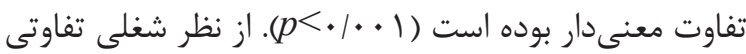

يرسيد سنجيده شد و بار ديخر، سنجش و ارزيابى حمايت

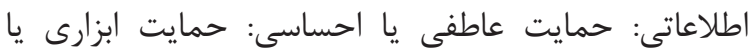

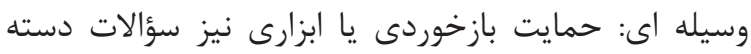

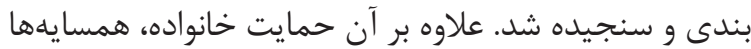

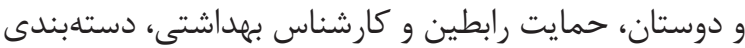
شده و تجزيه و تحليل شمان شد در اين مداخله، ابزارى به نام كام شمار براى اندازهخيرى،

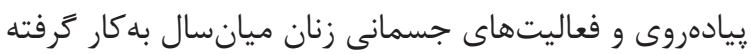

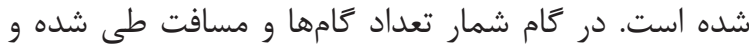

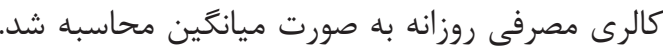

در مطالعههاى قبلى درباره وضعيت فعاليت جسمانى زنان

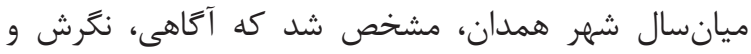

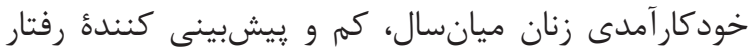

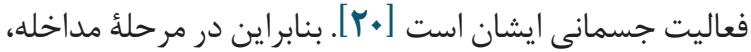

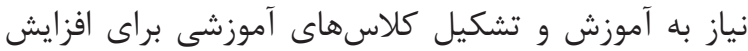

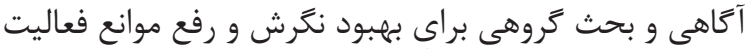

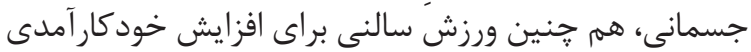

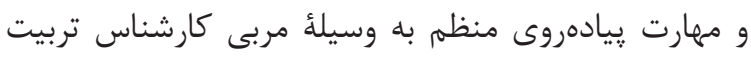

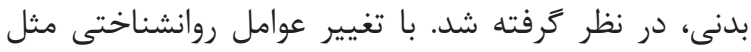

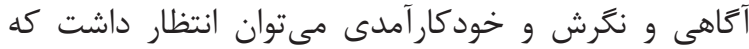

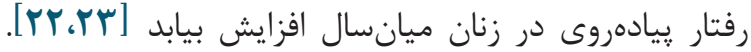

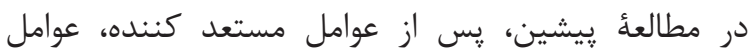

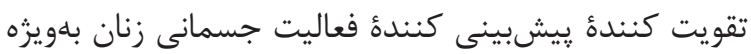

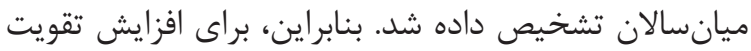

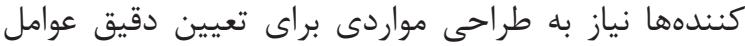

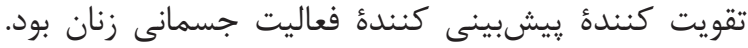
״س با تشكيل شبكة اجتماعى ميان زنان شركت كت كنندهاى كه

جدول ا. ميانغين و انحراف معيار نمرات تراز شده از •.ا، سازههاى مدل پرسيد در دوكروه مداخله و كنترل(قبل و سه ماه بعد از مداخله)

\begin{tabular}{|c|c|c|c|c|}
\hline \multirow{2}{*}{$\begin{array}{r}\text { p-value } \\
\text { Paired-test }\end{array}$} & بعد از مداخله & قبل از مداخله & \multirow{2}{*}{ كروه } & \multirow{2}{*}{ متغير } \\
\hline & mean \pm sd & $\operatorname{mean} \pm$ sd & & \\
\hline$<\cdot / \cdot \cdot 1$ & $\Lambda \Delta / F \cdot \pm I \cdot / N r$ & $r \cdot 19 \Lambda \pm r r / 9 r$ & مداخله & \multirow{3}{*}{ آكاهى } \\
\hline \multirow[t]{2}{*}{$<\cdot|\cdot|$} & VF/TGETr/Tr & $G 1 /{ }^{\prime} \Psi \Psi \Delta / r V$ & كنترل & \\
\hline & $*<+1 \cdot \cdot 1$ & $<\cdot \mid \cdot \cdot 1$ & $\begin{array}{r}\text { p-value } \\
\text { t-test }\end{array}$ & \\
\hline$<\cdot / \cdot \cdot 1$ & 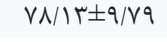 & $q N / F| \pm| F / \cdot q$ & مداخله & \multirow{3}{*}{ نعرش } \\
\hline \multirow[t]{2}{*}{.1191} & $\vee / / \Lambda V \pm / \varepsilon / \Delta \Lambda$ & 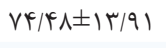 & كنترل & \\
\hline &.$/ .14$ & .1 .19 & $\begin{array}{r}\text { p-value } \\
\text { t-test }\end{array}$ & \\
\hline$<\cdot \mid \cdot \cdot 1$ & $q \pi / r \wedge \pm 1 \cdot / 9 \Delta$ & $r G / \Lambda \Lambda \pm I V / r \Lambda$ & مداخله & \multirow{3}{*}{ خودكار آمدى } \\
\hline \multirow[t]{2}{*}{ - IFFF } & $F r / T Y \pm T I / T r$ & $F F / 1 \cdot \pm 1 q / \Delta r$ & كنترل & \\
\hline & $*<\cdot 1 \cdot \cdot 1$ & & $\begin{array}{r}\text { p-value } \\
\text { t-test }\end{array}$ & \\
\hline$<\cdot \mid \cdot \cdot 1$ & $q \Gamma / q) \pm 11 / \vee q$ & $\uparrow \Delta / V \Delta \pm \mid \Delta / q$. & مداخله & \multirow{3}{*}{ تقويت كننده } \\
\hline \multirow[t]{2}{*}{.1 .14} & 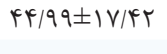 & $\Delta \| / / T^{\prime} \mid F^{\prime} / V^{f}$ & كنترل & \\
\hline & $<\cdot 1 \cdot \cdot 1$ & $\cdot / \cdot \Delta \Delta$ & $\begin{array}{r}\text { p-value } \\
\text { t-test }\end{array}$ & \\
\hline$\cdot / \cdot v$ & $V K / \Lambda \mu \pm 1 \cdot / K G$ & $99|\Delta| \pm|\varepsilon| \cdot 1$ & مداخله & \multirow{3}{*}{ قادر كننده } \\
\hline$\cdot / T \Delta T$ & $9 V / r r \pm 11 / 9 \pi$ & $99 / \cdot \uparrow \pm \mid r / \cdot \Lambda$ & كنترل & \\
\hline & $\cdot \cdot \cdot V$ & $\cdot / \Lambda 9$. & $\begin{array}{r}\mathrm{p} \text {-value } \\
\mathrm{t} \text {-test } \\
\end{array}$ & \\
\hline
\end{tabular}

مقادير p-value محاسبه شده بر مبناى تحليل كوواريانس است. 
جدول r. ميانَين و انحراف معيار نمرات حمايت اجتماعى تراز شده از •.1 ( اطلاعاتى، احساسى، وسيلهاى، بازخوردى) در دوكروه مداخله و

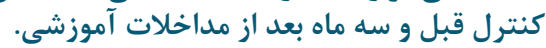

\begin{tabular}{|c|c|c|c|c|}
\hline $\begin{array}{r}\text { p-value } \\
\text { Paired t-test }\end{array}$ & $\begin{array}{r}\text { بعد از سه ماه مداخله } \\
\text { mean } \pm \text { sd }\end{array}$ & $\begin{array}{r}\text { قبل از مداخله } \\
\text { mean } \pm \text { sd }\end{array}$ & تروه - ت ك & حمايت اجتماعى \\
\hline$<\cdot|\cdot|$ & $\varepsilon T / \backslash G \pm V / 4 \varphi$ & $\langle\psi / R \xi \pm I Q / \Lambda D$ & كروه مداخله & \multirow{3}{*}{ حمايت اطلاعاتى } \\
\hline \multirow[t]{2}{*}{$\cdot 11}$. & 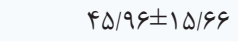 & $\Delta \cdot / \cdot 9 \pm 1 \Delta / r \Lambda$ & كروه كنترل & \\
\hline & $*<\cdot \mid \cdot \cdot 1$ &.$/ \cdot$ Ft & $\begin{array}{r}\mathrm{p} \text {-value } \\
\text { (t-test) }\end{array}$ & \\
\hline .1 .49 & RT/VTEIQ/IV & $r \Delta / \Delta \Delta \pm / N / q \gamma$ & كروه مداخله & \multirow{3}{*}{ حمايت ابزارى } \\
\hline \multirow[t]{2}{*}{$\cdot 1 \cdot \Delta$} & $r g / V \Psi \pm \mid g / Q F$ & $M Y / G Y \pm I V / \Lambda F$ & كروه كنترل & \\
\hline & $<\cdot|\cdot \cdot|$ & •/VAT & $\begin{array}{r}\text { p-value } \\
\text { (t-test) }\end{array}$ & \\
\hline$<\cdot 1 \cdot \cdot 1$ & $\vee ৭ / \Delta ৭ \pm \wedge / ৭ \varphi$ & $\Delta N / 1 / \pm 19 / \cdot r$ & كروه مداخله & \multirow{3}{*}{ حمايت احساسى } \\
\hline \multirow[t]{2}{*}{$\cdot 11}$. & $9 \cdot / 19 \pm 1 \Lambda / \Lambda$. & $\varphi Q / \cdot \Lambda \pm \mid \Lambda / Y F$ & \multirow{2}{*}{ 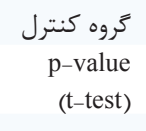 } & \\
\hline & $*<\cdot \mid \cdot 1$ &.$/ \cdot F T$ & & \\
\hline$<\cdot|\cdot|$ & $V r / q V \pm I r / V q$ & $r \Delta / q \cdot \pm r \cdot / q V$ & كروه مداخله & \multirow{3}{*}{ حمايت بازخوردى } \\
\hline \multirow[t]{2}{*}{.119} & $F V / \wedge \Delta \pm T V / V F$ & $\Delta r / \Delta q \pm r / / \Delta r$ & \multirow{2}{*}{ 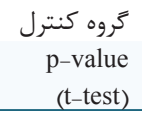 } & \\
\hline & $*<\cdot / \cdots 1$ & $\cdot / \cdot \uparrow \wedge$ & & \\
\hline
\end{tabular}

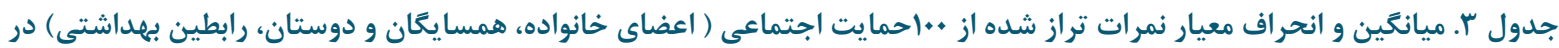

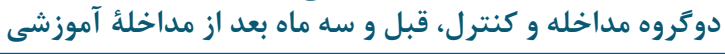

\begin{tabular}{|c|c|c|c|c|}
\hline $\begin{array}{r}\text { p-value } \\
\text { Paired t-test }\end{array}$ & 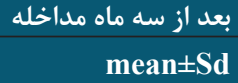 & $\begin{array}{l}\text { قبل مداخله } \\
\text { mean } \pm \text { Sd }\end{array}$ & تروه & حمايت اجتماعى \\
\hline$<\cdot|\cdot|$ & $4 \cdot|\varphi \Delta \pm| f / v \Delta$ & FN/ATEIN/II & مداخله & \multirow[b]{2}{*}{ اعضاى خانواده } \\
\hline$\cdot / l \Delta V$ & $\begin{aligned}\forall N / q) & \pm r \mid / r . \\
& <\cdot / \cdot \cdot 1\end{aligned}$ & $\begin{array}{r}\Delta T / F \cdot \pm \mid \varepsilon / \Delta V \\
\cdot / T \Delta S\end{array}$ & $\begin{array}{r}\text { كنترل } \\
\text { p-value } \\
\text { (t-test) }\end{array}$ & \\
\hline$<\cdot 1 \cdot \cdot 1$ & $\Delta N / \cdot \Lambda \pm I \Delta / V \varphi$ & 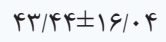 & مداخله & \multirow[b]{2}{*}{ همسايعان } \\
\hline $.1 \cdot 1$ & $\begin{array}{r}r q / \Delta r \pm \mid Q / \cdot \wedge \\
*<\cdot / \cdot \cdot 1\end{array}$ & $\begin{array}{r}r q / .9 \pm 18 / 19 \\
. / .4 q\end{array}$ & $\begin{array}{r}\text { كنترل } \\
\text { p-value } \\
\text { (t-test) }\end{array}$ & \\
\hline$<\cdot / \cdot \cdot 1$ & $\Lambda \cdot / \xi \uparrow \pm 11 / 9 \Delta$ & $\uparrow r / \varnothing \uparrow \pm r \mid / \Delta \Lambda$ & مداخله & \multirow[b]{2}{*}{ بهربى و رابطان } \\
\hline$\cdot / \pi \mid \Lambda$ & $\begin{array}{r}F V / r F \pm T V / \cdot q \\
*<\cdot / \cdot \cdot 1\end{array}$ & $\begin{array}{r}\Delta T / / \Delta \pm T Y / q T \\
\cdot / \cdot r \Delta\end{array}$ & $\begin{array}{r}\text { كنترل } \\
\text { p-value } \\
\text { (t-test) }\end{array}$ & \\
\hline
\end{tabular}

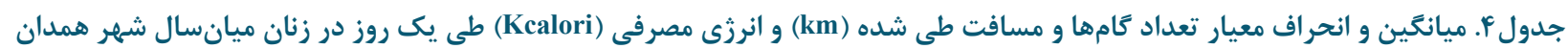

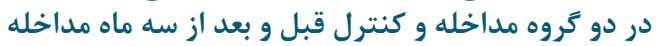

\begin{tabular}{|c|c|c|c|c|}
\hline $\begin{array}{r}\text { p-value } \\
\text { Paired-test }\end{array}$ & $\begin{array}{r}\text { سه ماه بعد از مداخله } \\
\text { mean } \pm \text { SD }\end{array}$ & $\begin{array}{r}\text { قبل از مداخله } \\
\text { mean } \pm \text { SD }\end{array}$ & تروه & متغير \\
\hline$\cdot 1 \cdot 1$ & $V r F V / q r \pm r V M \Lambda / \cdot r$ & $q 1 r \cdot / F q \pm r \Lambda \Delta \Lambda / r F$ & مداخله & \multirow{3}{*}{ تعداد كَامها } \\
\hline$<\cdot / \cdot \cdot 1$ & $r q \gamma r / \Delta) \pm r \Delta \cdot r / \Delta q$ & 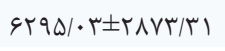 & كنترل & \\
\hline & $<\cdot \cdot \cdot \cdot 1$ & - VQT & $\begin{array}{r}\mathrm{p} \text {-value } \\
\mathrm{t} \text {-test }\end{array}$ & \\
\hline$\cdot 1 \cdot \Delta$ & $r V Q / T r \pm \mid F \cdot / r \Lambda$ & $r T \Lambda / q \vee \pm 1 \cdot G / T 1$ & مداخله & \multirow{3}{*}{$\begin{array}{r}\text { انرزى مصرفى } \\
\text { kcalori }\end{array}$} \\
\hline$<\cdot / \cdot \cdot 1$ & $\mid \Lambda \Delta / \Delta \vee \pm q r / \mathbb{A} \Delta$ & $r \mu r / V G \pm 9 \Delta / V$ & كنترل & \\
\hline & $<\cdot|\cdot|$ & • & $\begin{array}{r}\text { p-value } \\
\text { t-test }\end{array}$ & \\
\hline.$/ \cdot r$ & $|f / f| \pm 1 / \Lambda \varepsilon$ & $r / 9 \wedge \pm 1 / V r$ & مداخله & \multirow{3}{*}{ مسافت طى شده } \\
\hline \multirow[t]{2}{*}{$<\cdot / \cdot \cdot 1$} & $r / Q \wedge \pm 1 / \Delta$. & $r / v \wedge \pm 1 / \varphi$. & كنترل & \\
\hline & $<\cdot / \cdot \cdot 1$ & - $/ A T A$ & $\begin{array}{r}\mathrm{p} \text {-value } \\
\mathrm{t} \text {-test }\end{array}$ & \\
\hline
\end{tabular}




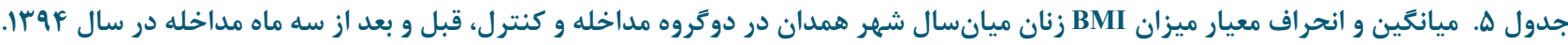

\begin{tabular}{|c|c|c|c|}
\hline \multirow{2}{*}{$\begin{array}{c}\text { p-value } \\
\text { Paired t-test }\end{array}$} & BMI بعد از مداخله & BMI قبل از مداخله & \multirow[t]{2}{*}{ كروه } \\
\hline & $\operatorname{mean} \pm$ sd & $\operatorname{mean} \pm$ sd & \\
\hline$<\cdot 1 \cdot \cdot 1$ & $r V / q \Delta \pm F / V I$ & $\Gamma \wedge / \Delta S \pm Y / \Lambda \varphi$ & مداخله \\
\hline \multirow[t]{2}{*}{.1 .91} & KN/GDET/Tr & $r \Lambda / F \mid \pm r / \varphi$. & كنترل \\
\hline & - MFF & - & $\begin{array}{r}\mathbf{p} \text {-value } \\
\text { t-test }\end{array}$ \\
\hline
\end{tabular}

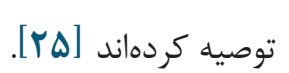

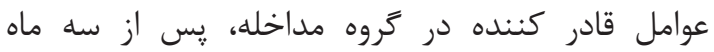

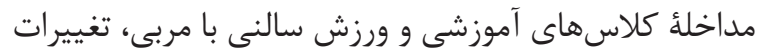

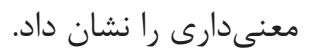

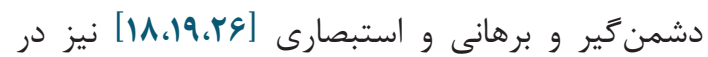

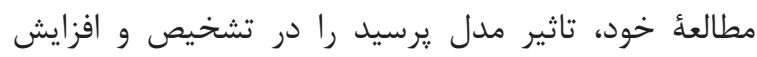

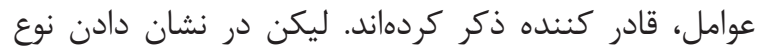

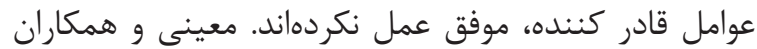

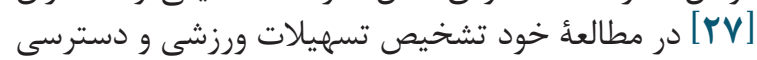

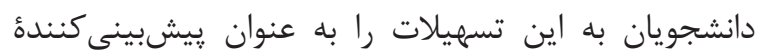

$$
\text { فعاليت جسمانى ايشان نام مىبرد. }
$$

استفاده از مدل زيرسيد و حمايت اجتماعى با تشكيل

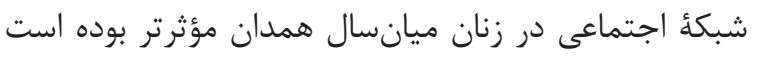

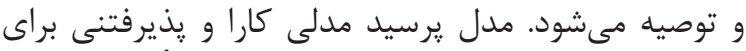

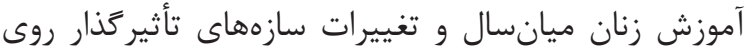
رفتار فعاليت جسمانى ايشان است

رفتار فعاليت جسمانى مناسب براى ميانسالان، ييادهروى ترى

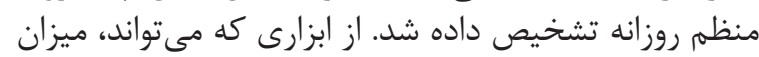

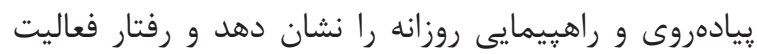

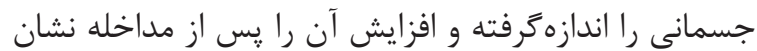
دهد يعنى كام شمار استفاده شد.

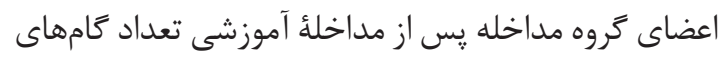

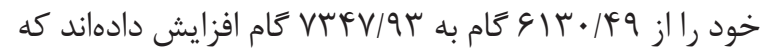

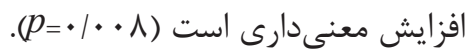

درٍ اين گرووه مسافت طلى شده نيز يس از مداخلة آموزشى،

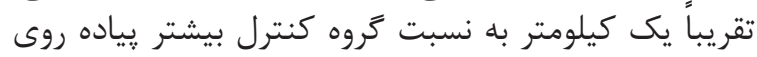

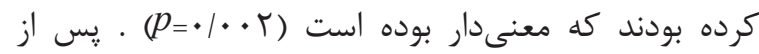

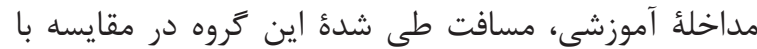

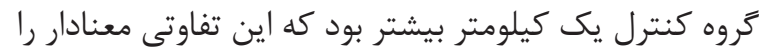

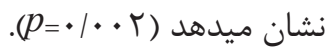

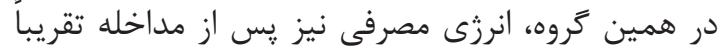

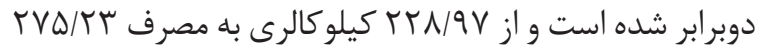

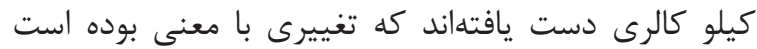

$$
\cdot\left(p_{=} \cdot / \cdot \cdot \Delta\right)
$$

شاخص تودهُ بدنى به عنوان رييامدى براى رفتار فعاليت

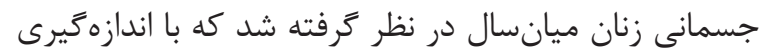

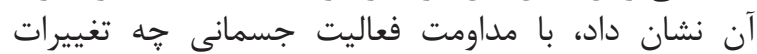

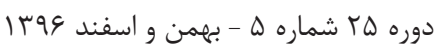

مشاهده نشده و خانهدارها بيشترين نفرات هر دو زروه را

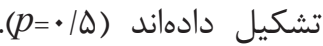

\section{بحث}

در اين مطالعه، در سازههاى مستعدكننده آكَاهى و نكَرش

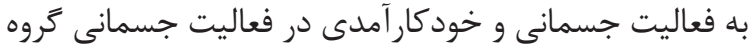

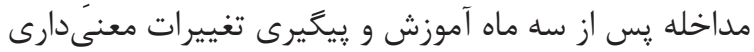

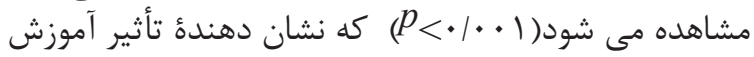

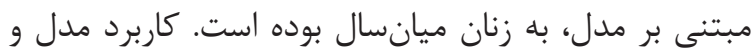

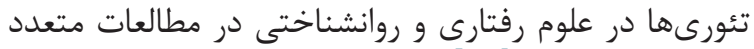

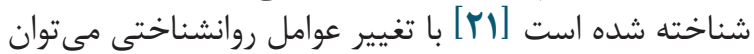

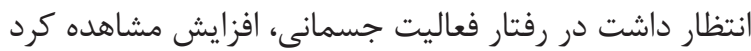

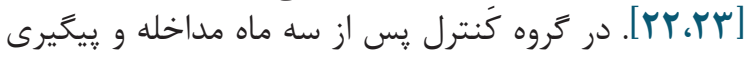

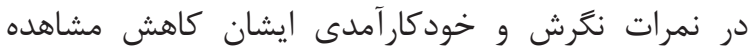

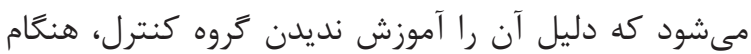

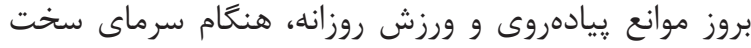

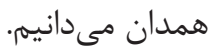

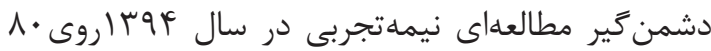

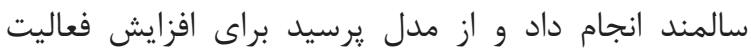

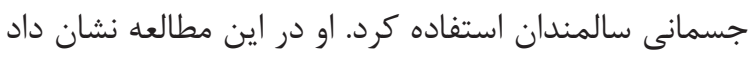

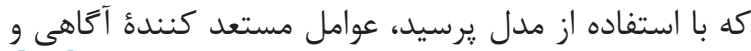

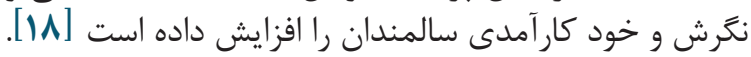

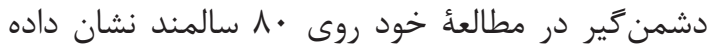

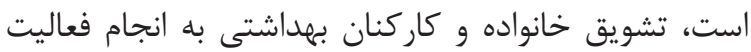

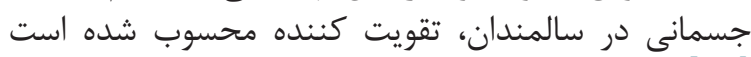

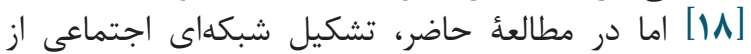

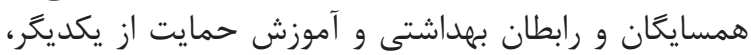

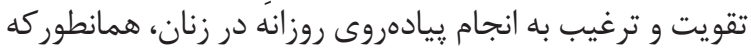

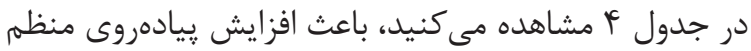
روزانه در زروه مداخله شده است. مدهد مئدي

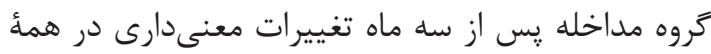

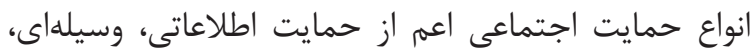

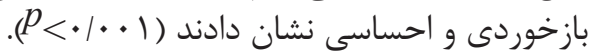

كاواكى و همكاران در مطالعئ خود، داشتن شبكئ اجتماعى

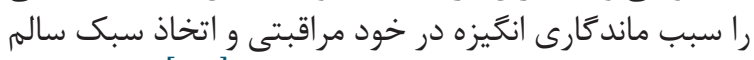

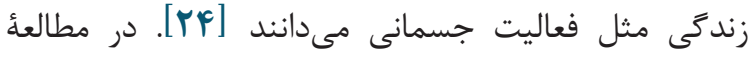

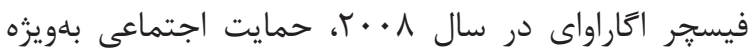

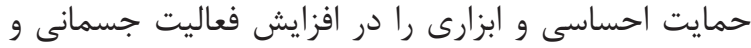

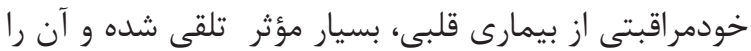
مجله علمى دانشكده يرستارى و مامايى همدان 


\section{نتيجه}

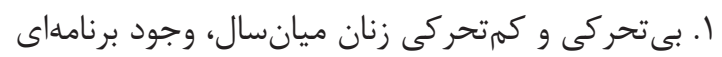

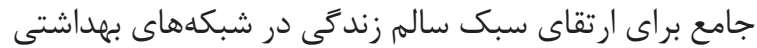

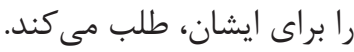

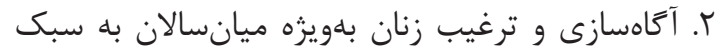

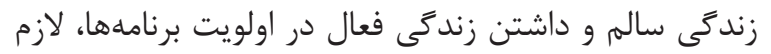

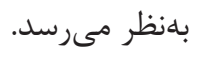

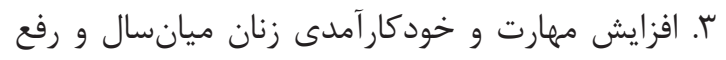

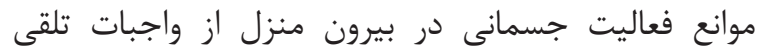
مى شود.

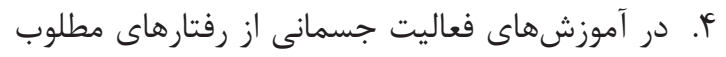
اجرايى و مقبول براى ميانسالان مثل يُ يادهروى استفاده

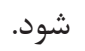

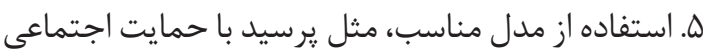

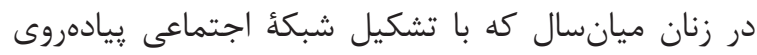
روزانه صورت كرفت، براى ايجاد انكَيزه و ترغيب آنان بنان لازم

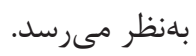

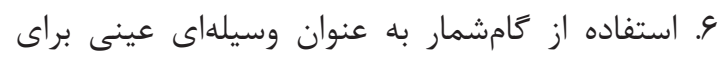
سنجش فعاليت جسمانى يُشنهار ازيهاد مى شود.

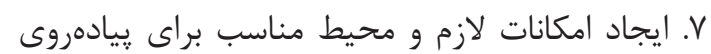

$$
\text { ميانسالان توصيه مى إنود. }
$$

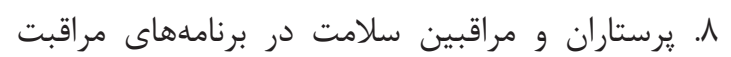

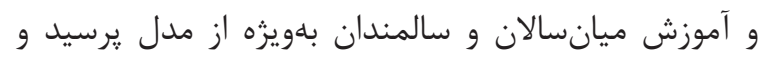
حمايت اجتماعى در زنان استفاده كنند.

\section{سياسگزارى}

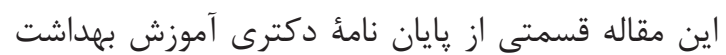

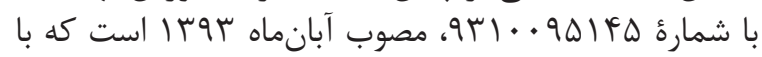

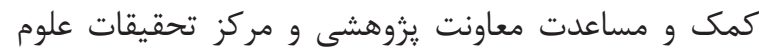

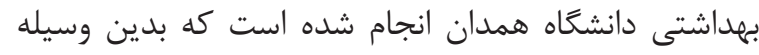

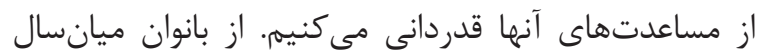

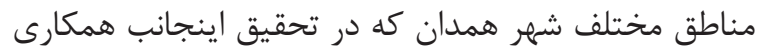

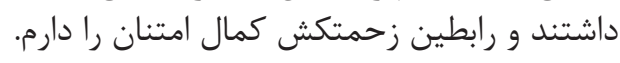

$$
\text { تعارض در منافع }
$$

بين نويسندكان در اين مطالعه تضاد منافعى وجود ندارد.
קشمخيرى مىتوان انتظار داشت. جدول ه نشان مىدهد

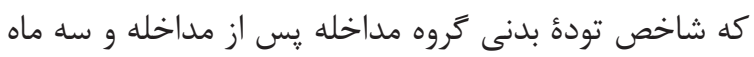

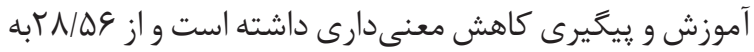

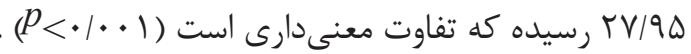

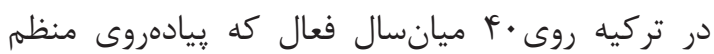

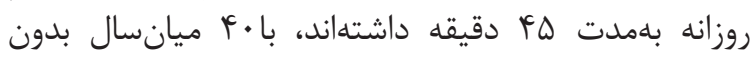

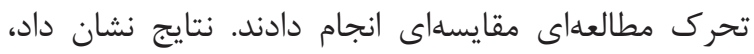

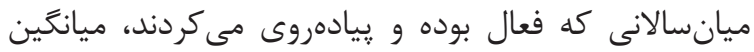

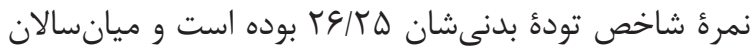

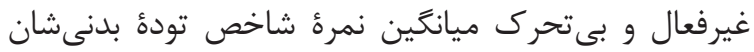

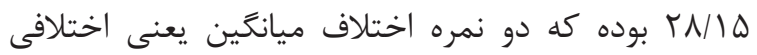

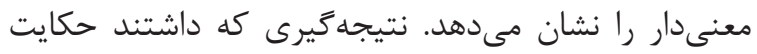

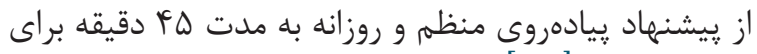

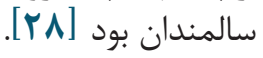

$$
\text { نتيجه }
$$

اين مطالعه پِّ از تحليل فعاليت جسمانى زنان ميانسال

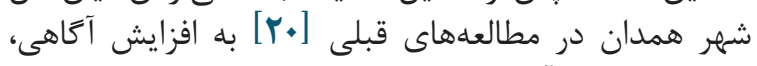

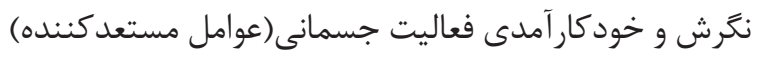

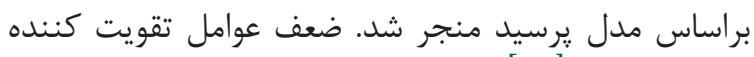

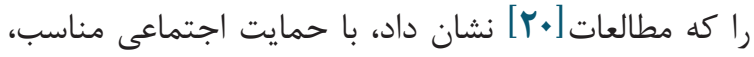

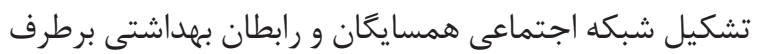

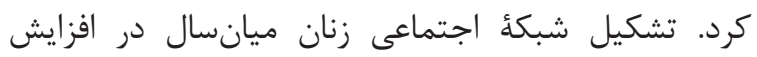

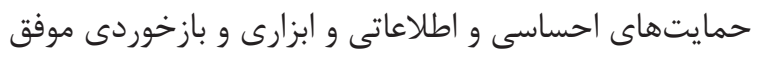

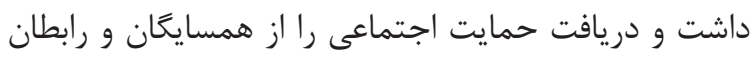

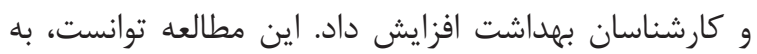

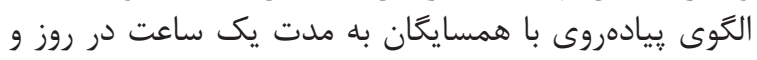

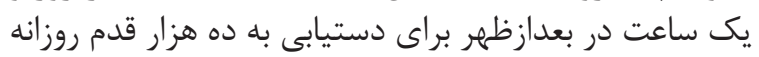

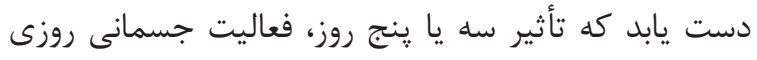

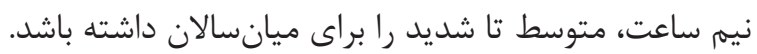

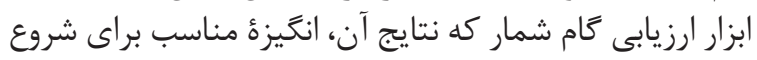

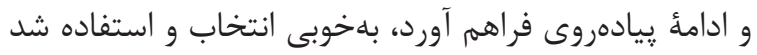

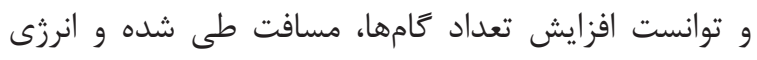

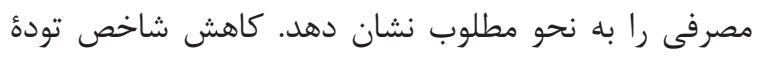

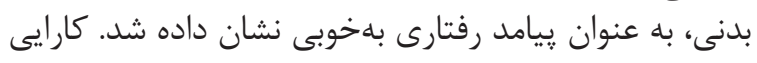

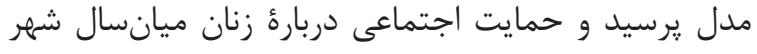

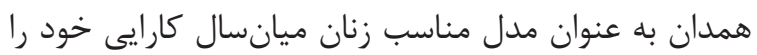

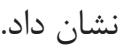




\section{References}

1. Tumminello M, Micciche S. Dominguez L J, Lamura G, Melchiorre MC, Barbagallo M, et al. Happy aged people are all alike, while every unhappy aged persons is unhappy in its own way. PloS One. 2011;6(9):e23377. https://doi.org/10.1371/ journal.pone.0023377 PMid:21931596 PMCid:PMC3169534

2. De Souza Santos CA, Dantas EE. Moreira MH. Correlation of physical aptitude: functional capacity, corporal balance and quality of life(QoL) among elderly women submitted to a post-menopausal physical activities program. Arch Gerontol Geriatr. 2011;53(3):344-9. https://doi.org/10.1016/j.archger.2010.12.019 PMid:21288578

3. Hadi N, Hajjari Z. Women's health in Iran: A Review. Shiraz E-Medical Journal. 2013;14(3):17290.

4. Gretebeck RJ, Ferraro KF, Black DR, Holland $\mathrm{K}$, Gretebeck KA. Longitudinal change in physical activities in adults. Am J Behav. 2012;36(30):385-94.

5. Wipfli BM, Rethorst CD, Landers DM. The anxiolytic effects of exercise: a meta analysis of randomized trials and dose-response analysis. J Sport Exerc psycho. 2008;30(4):392-410. https://doi.org/10.1123/jsep.30.4.392

6. Lianas AC, Hachul H, Bittencourt LR, Tufik S. Physical therapy reduces insomnia symptoms in postmenopausal women. Maturitas. 2008;61(3):281-4. https://doi.org/10.1016/j.maturitas.2008.08.004 PMid: 18819761

7. de Azevedo Guimaraes AC, Baptisa F. Influence of habitual physical activity on the symptoms of climacterium / menopause and the quality of life if middle-aged women. Int $\mathrm{J}$ Womens Health. 2011;3:319-28. https://doi.org/10.2147/IJWH. S24822 PMid:22114524 PMCid:PMC3220314

8. Baer HJ, Glynn RJ, Hu FB, Hankinson SE, Willett WC, Colditz GA, et al. Risk factors for mortality in the nurses' health study: a competing risks analysis. Am J Epidemiol. 2011;173(3):319-29. https://doi.org/10.1093/aje/ kwq368 PMid:21135028 PMCid:PMC3105270

9. Barg CJ, Latimer AE, Pomery EA, Rivees SE, Rench TA, Prapavessis H, Salovey P. Examining predictors of physical activity among inactive middle-aged women: An application of the health action process approach. Psychol Health.2012;27(7):829-45. https://doi.org/10.10 80/08870446.2011.609595 PMid:21867395 PMCid:PMC3288881

10. Rolland Y,Abellan van kan G, Vellas B. Healthy Brain Aging: Role of exercise and physical activity. Clin Geriatr Med. 2010;26(1):75-87. https://doi.org/10.1016/j.cger.2009.11.002 PMid:20176294

11. Eliassen AH, Hankinson SE, Rosner B, Holmes MD, Willett WC. Physical activity and risk of breast cancer among postmenopausal women. Arch Intern Med. 2010;170(19):1758-64. https://doi.org/10.1001/archinternmed.2010.363 PMid:20975025 PMCid:PMC3142573

12. Javadivala $Z$, Kousha A, Allahverdipour $\mathrm{H}$, Asghari Jafarabadi M, Tallebian H. Modeling the relationship between Physical Activity and Quality of Life in Menopausal-aged Women:A cross-sectional Study. J Res Health Sci. 2013;13(2):168-75. PMid:24077475

13. Daley A, MacArthur C, Stokes-Lampard H, McManus R, Wilson S, Mutrie N. Exercise participation, body mass index, and health-related quality of life in women of menopausal age. $\mathrm{Br} \mathrm{J}$ Gen Pract. 2007;57(535):130-5. PMid: 17266830 PMCid:PMC2034173

14. Belza B, Warms C. Physical activity and exercise in women's health. Nurs Clin North Am. 2004;39(1):181-93. https://doi.org/10.1016/j. cnur.2003.11.011 PMid:15062735

15. WHO. Insufficient physical activity 2010. Prevalence of insufficient physical activity among adults $=$ ages $18+$ (age standardized estimates ).who.org.

16. Koohpayehzadeh J, Etemad K, Abbasi M, Meysamie A, Sheikhbahaei S, Asgari F, et al.Gender-specific changes in physical activity pattern in Iran:national surveillance of risk factors of non-communicable diseases(2007-2011). Int J Public Health. 2014;59(2):231-41. https://doi.org/10.1007/s00038-013-0529-3 PMid:24346180

17. Glanz k, Rimer BK, Lewis FM. Health behavior and Health education : Theory, Research and practice.3nd ed. New Jersey: Jossey-Bass; 2002. p:411-45.

18. Doshmangir P, Shirzadi SH, Tagdisi MH, Doshmangir L. The effect of educational intervantion according to PRECEDE model on the regular physical activity in elderly people. J Educ Community Health. 2014;1(2):1-9. https://doi. org/10.20286/jech-01021

19. Dizaji MB, Taghdisi MH, Solhi M, Hoseini SM, Shafieyan Z, Qorbani M, et al. Effects of educational intervention based on PRECEDE model on self care behaviors and control in patients with type 2 diabetes in 2012. J Diabetes Metab Disord. 2014;13(1):72. https://doi. org/10.1186/2251-6581-13-72 PMid:25075380 PMCid:PMC4114427

20. Emdadi SH, Hazavehei SMM, Soltanian A, 
Bashirian S, Heidari Moghadam R. Predictive Factors of Regular Physical Activity among Middle-aged Women in West of Iran Hamadan: Application of PRECEDE Model. J Res Health Sci. 2015;15(4):244-9. PMid:26728911

21. Glanz K, Bishop DB. The role of behavioral science theory in development and implementation of public health interventions. Annu Rev Public Health. 2012;31:399-419. https://doi. org/10.1146/annurev.publhealth.012809.103604 PMid:20070207

22. Todd J, Mullan B. Using the theory of planned behavior and prototype willingness model to target binge drinking in female undergraduate university students. Addict Behav. 2011;36(10):9806. https://doi.org/10.1016/j.addbeh.2011.05.010 PMid:21669494

23. Barati M, Allahverdipour H, Hidarnia A, Niknami S, Belief-based Tabacco Smoking Scale: Evalutiuating the psychometric Properties of the Theory of Planned Behavior's Constructs. Health Promt Perspect. 2015;5(1):59-71. https://doi. org/10.15171/hpp.2015.008 PMid:26000247 PMCid:PMC4430699

$$
\text { 19 ا. بررسى تأثير آموزش مبتنى بر مدل بر فعاليت ... }
$$

24. Kawachi I, Berkman LF. Social ties and mental health. J Urban Health. 2001;78(30):458-67. PMCid:PMC3455910

25. Fischer Aggarwai BA, Liao M, Mosca L. Physical activity as potential mechanism through which socialsupport may reduce cardiovascular disease risk. J cardiovasc Nurs. 2008;23(2):90-6. PMid: 18382248 PMCid:PMC2674263

26. Estebsari F, Shojaeizadeh D, Mostafaei D, Farahbakhsh M. Planning and Evalution of an Educational Program Based on PRECEDE Model to Improve Physical Activity in Female Students . HAYAT. 2010;16(1):48-54.

27. Moeini B, J. F., Jallilian M, Barati M. 2011. Predicting factors associated with Regular activity among college students applying BASNEF model. Avicenna J Clin Med. 2011;18(3):70-6.

28. Atalag O, Cavlak U. The impact of unsupervised regular walking on health: A sample of Turkish middle-aged and older Adult. Eur Rev Aging Phys Act. 2012;9(1);71-9. https://doi. org/10.1007/s 11556-011-0083-Z 